\title{
Mysterious Abdominal Pain
}

\author{
Alberto Cappelletti, M.D.,' ${ }^{1}$ Nicole Cristell, M.D., ${ }^{1}$ Monica Mazzavillani, M.D., ${ }^{1}$ \\ and Alberto Margonato, M.D., F.E.S.C. ${ }^{1}$
}

A man presented to the emergency room with recurrent episodes of abdominal pain. He had a history of coronary artery bypass grafting of the left internal mammary artery (LIMA) to the left anterior descending (LAD) artery and the right gastroepiploic artery to the posterior descending artery. After numerous gastrointestinal evaluations, a stress test was performed, which was positive. Coronary angiography showed a proximal occlusion of the LAD and right coronary artery and a normal functioning LIMA bypass. Aortography showed a 95\% stenosis of the celiac trunk. Angioplasty and stent implantation of the celiac trunk was successfully performed. Six months later the patient was completely asymptomatic with a negative stress test. In conclusion, abdominal pain in patients who have undergone coronary artery bypass surgery using the right gastroepiploic artery should raise suspicion not only of a stenosis of the arterial conduit but also of a potential stenosis of the celiac trunk.

KEYWORDS: Abdominal pain, coronary artery bypass grafting, right gastroepiploic artery, celiac trunk, angioplasty, stent implantation

Recurrent abdominal pain is a frequent reason for patients seeking medical attention and may have a large differential diagnosis. ${ }^{1,2}$ In this case report, we describe a patient with recurrent abdominal pain where the initial interpretation of the symptoms was difficult but the final outcome was positive.

\section{CASE REPORT}

A 54-year-old man with hypertension and dyslipidemia presented to the emergency room with recurrent episodes of abdominal pain. He had a history of coronary artery bypass grafting of the left internal mammary artery (LIMA) to the left anterior descending artery (LAD) and the right gastroepiploic artery to the posterior descending artery.

Clinical examination and basic blood work-up were normal. An abdominal ultrasound was performed because of a recent episode of pancreatitis. Gallstones were visualized in the gallbladder resulting in hospital admission and subsequent elective cholecystectomy. Unfortunately, his symptoms did not subside and thus a gastroscopy was performed that showed the presence of a hiatus hernia associated with initial signs of esophagitis but no gastric or duodenal pathologies. A breath test was also performed, confirming the presence of Helicobacter pylori, which was treated with omeprazole and amoxicillin for a month with subsequent confirmation of eradication.

As symptoms still persisted, a stress test was performed which was positive with $2 \mathrm{~mm} \mathrm{ST}$-segment depression in the inferior leads. He therefore underwent coronary angiography that showed a proximal occlusion of the LAD and right coronary artery and a normal functioning LIMA bypass. During the same procedure, an abdominal aortogram was performed to visualize the major abdominal arteries and demonstrated a $95 \%$ stenosis of the celiac trunk (Fig. 1).
${ }^{1}$ Department of Cardiology, San Raffaele Scientific Institute, Milan, Italy.

Address for correspondence and reprint requests: Alberto Cappelletti, M.D., Department of Cardiology, San Raffaele Scientific Institute, Via Olgettina 60, 20132 Milan, Italy (e-mail: albertomariacappelletti@gmail.com).
Int J Angiol 2011;20:181-184. Copyright (C) 2011 by Thieme Medical Publishers, Inc., 333 Seventh Avenue, New York, NY 10001, USA. Tel: +1(212) 584-4662.

Published online: July 18, 2011.

DOI: http://dx.doi.org/10.1055/s-0031-1284202.

ISSN 1061-1711. 


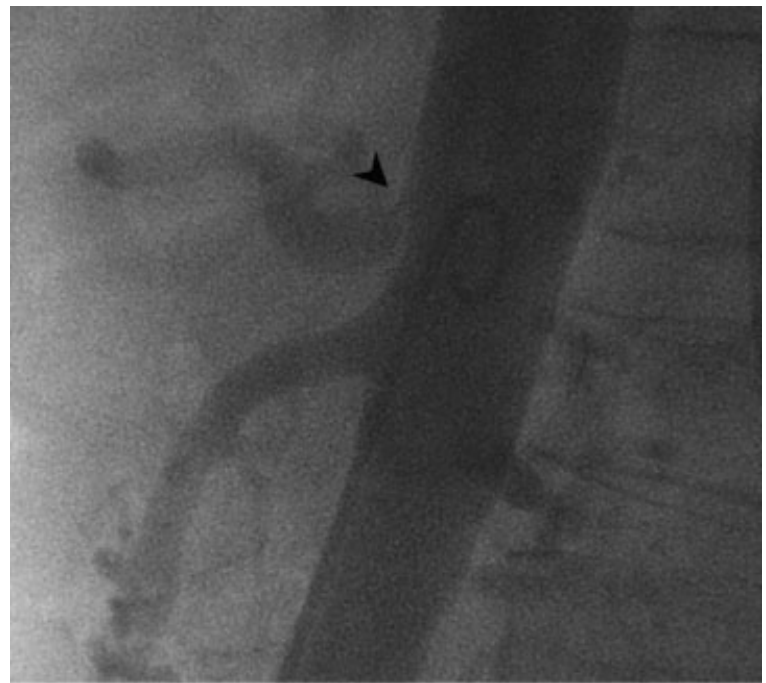

Figure 1 Abdominal angiography (left lateral view) showing severe stenosis of the celiac trunk (arrow).

We decided to treat the lesion percutaneously. A $7 \mathrm{Fr}$ renal catheter was positioned and the lesion was successfully stented with a $7.0 \times 15 \mathrm{~mm}$ Hippocampus stent implanted at 10 atmospheres. The immediate angiographic result was excellent (Fig. 2). Six months later, the patient was completely asymptomatic with a negative stress test.

\section{DISCUSSION}

It is well known that upper abdominal pain is a frequent symptom of many benign gastrointestinal pathologies and often patients who have undergone coronary artery bypass surgery using the right gastroepiploic artery can have a normal clinical examination and blood tests. ${ }^{1,2}$ Thus, reaching a definitive diagnosis can be quite challenging. Often patients who have aspecific abdominal symptoms are classified as having functional disorders

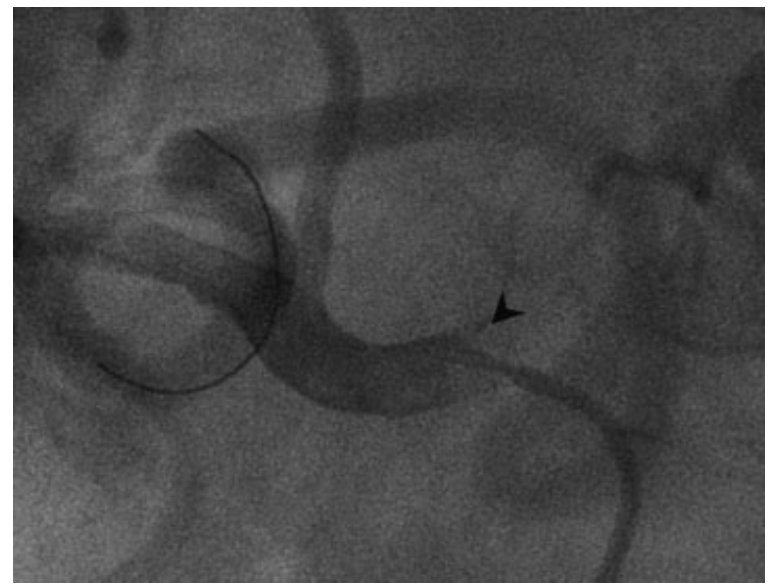

Figure 2 Angiographic result at the end of the procedure (arrow). such as gastroesophageal reflux, ${ }^{3}$ psychosomatic gastritis, ${ }^{4}$ irritable bowel syndrome, and generic dyspepsia without organic pathologies. ${ }^{5}$

Celiac trunk stenosis is a rare pathology and, to the best of our knowledge, successful treatment of myocardial ischemia secondary to this stenosis has not been previously described. The only similar reports in the literature do not describe treatment of the celiac trunk but percutaneous angioplasty of the gastroepiploic bypass graft, ${ }^{6}$ or of the hepatic artery to treat coronary steal. ${ }^{7}$ Arterial conduits have become the most frequently used and preferred grafts during coronary artery bypass surgery. The internal mammary arteries are the first choice; ${ }^{8}$ radial arteries ${ }^{9}$ and the right gastroepiploic $\operatorname{artery}^{10}$ are also used albeit less frequently. The gastroepiploic artery has been more frequently used in Europe as a conduit to bypass the right coronary artery. ${ }^{11}$ It originates from the gastroduodenal artery, which itself arises from the hepatic artery, which in turn is one of the three main branches of the celiac trunk. In our case, the patient had a LIMA on the LAD and the right gastroepiploic artery on the posterior descending artery. His symptoms and the positive stress test were secondary to ischemia of the inferior wall due to the celiac trunk stenosis and subsequent hypoperfusion of the gastroepiploic artery bypass graft. Proof of this concept is the complete symptom regression as well as a negative stress test after celiac trunk stent implantation.

In conclusion, abdominal pain in patients who have undergone coronary artery bypass surgery using the right gastroepiploic artery should raise suspicion of not only a stenosis of the arterial conduit but also a potential stenosis of the celiac trunk.

\section{REFERENCES}

1. Heading RC. Prevalence of upper gastrointestinal symptoms in the general population: a systematic review. Scand J Gastroenterol Suppl 1999;231:3-8

2. Matsunaga S, Eguchi Y. Importance of a physical examination for efficient differential diagnosis of abdominal pain: diagnostic usefulness of Carnett's test in psychogenic abdominal pain. Intern Med 2011;50(3):177-178

3. Vaezi MF. Reflux monitoring: on or off therapy? Am J Gastroenterol 2011;106(2):183-185

4. Loyd RA, McClellan DA. Update on the evaluation and management of functional dyspepsia. Am Fam Physician 2011;83(5):547-552

5. Chang JY, Talley NJ. An update on irritable bowel syndrome: from diagnosis to emerging therapies. Curr Opin Gastroenterol 2010;27(1):72-78

6. Roriz R, de Gevigney GD, Howarth N, Revel D. Case of successful percutaneous stenting of an in situ gastroepiploiccoronary bypass graft. Cathet Cardiovasc Diagn 1998;45(1): $67-69$

7. Kandzari DE. Catheter-based revascularization of the hepatic artery to treat coronary steal from a gastroepiploic artery bypass graft. J Invasive Cardiol 2003;15(10):591-593 
8. Kurlansky P. Thirty-year experience with bilateral internal thoracic artery grafting: where have we been and where are we going? World J Surg 2010;34(4):646-651

9. Tranbaugh RF, Dimitrova KR, Friedmann P, et al. Radial artery conduits improve long-term survival after coronary artery bypass grafting. Ann Thorac Surg 2010;90(4):11651172
10. Suma H, Tanabe $H$, Takahashi A, et al. Twenty years experience with the gastroepiploic artery graft for CABG. Circulation 2007;116(11, Suppl):I188-I191

11. Ali E, Saso S, Ashrafian H, Athanasiou T. Does a skeletonized or pedicled right gastro-epiploic artery improve patency when used as a conduit in coronary artery bypass graft surgery? Interact Cardiovasc Thorac Surg 2010;10(2): 293-298 